\title{
CAMBIOS EN LA DISTRIBUCIÓN DE LOS GENOTIPOS DEL VIRUS DE LA HEPATITIS C DURANTE EL PERIODO 1999-2010 EN EL ÁREA OESTE DE VALLADOLID
}

\author{
María del Carmen Ramos-Sánchez y Francisco Javier Martín-Gil.
}

Hospital Universitario Río Hortega. Valladolid.

\begin{abstract}
RESUMEN
Fundamento: Se realizó un análisis retrospectivo de los resultados de genotipado en sueros de pacientes con hepatitis C remitidos a nuestro laboratorio del Hospital Universitario Río Hortega (Valladolid) durante el periodo 1999-2010. El motivo de esta investigación fue constatar, en nuestra área geográfica, la sugerencia de otros autores sobre la diseminación de las cepas del genotipo 4. El objetivo fue conocer la prevalencia de cada genotipo y compararlo con la prevalencia en el resto del país.
\end{abstract}

Métodos: El número de pacientes estudiados fue de 1.074 La extracción de ARN del VHC se llevó a cabo mediante el sistema COBAS Ampli-Prep (Roche). La transcripción inversa, amplificación e hibridación inversa se realizaron con reactivos Qiagen y Siemens. En pacientes coinfectados VHC-VIH la prueba de anticuerpos anti-VIH se realizó por una técnica EIA y en caso de positividad fue confirmada por WesternBlot o LIA.

Resultados: El genotipo VHC más frecuente fue el 1 $(69 \%)$, seguido por el $3(19,6 \%)$ y el $4(8,2 \%)$. El subtipo VHC más frecuente fue el $1 \mathrm{~b}(41,3 \%)$. La coinfección VIH-VHC en los pacientes con genotipo 4 de nuestro medio geográfico alcanzó el 58\%. Desde el informe previo que realizamos en 2002 hasta la actualidad (finales de Diciembre 2010) los cambios producidos fueron un aumento de prevalencia para los genotipos 4 (del $7,3 \%$ al $8,8 \%), 1$ y 1 a (del $25,9 \%$ al $29,4 \%$ ) y una disminución para el $1 \mathrm{~b}$ (del $44 \%$ al 39,5\%). Los pacientes con genotipo 4 fueron, mayoritariamente, varones y con coinfección VIH-VHC.

Conclusiones: La prevalencia del genotipo 4 en el área estudiada fue significativamente diferente de la media estatal, lo que llevó a concluir que la dispersión de este genotipo es mucho más lenta de lo previsto.

Palabras clave: Hepatitis C. Genotipos. Dispersión.

\section{ABSTRACT}

\section{Changes in the Distribution of Hepatitis C Virus (HCV) Genotypes over the 1999- 2010 Period in Northern Valladolid, Spain}

Background: A retrospective analysis was carried out in all specimens from subjects with chronic hepatitis $\mathrm{C}$ sent for testing to our laboratory in Hospital Universitario Río Hortega (Valladolid, Spain) over the period 1999-2009. The reason for this study was to examine the suggestion of other authors on the spread of genotype 4 strains. The objective was to describe the distribution of VHC genotypes in our geographical area and compare it with other state-wide reports.

Methods: A total of 1074 patients were studied. Specimen preparation to isolate HCV RNA was carried out with the COBAS AmpliPrep system (Roche). Reverse transcription, amplification and reverse hybridization were performed with Qiagen and Siemens kits.

Results: The most frequent HCV genotype was $1(69 \%)$, followed by $3(19,6 \%)$ and $4(8.2 \%)$. The most frequent HCV subtype was $1 \mathrm{~b}(41.3 \%)$. Most infections with genotype 4 (58\%) were found among HCV-HIV-coinfected patients. From our previous report in 2002 to date (end December 2010), both an increase in the prevalence of genotypes 4 (from 7.3 to $8.8 \%$ ) and 1 and $1 \mathrm{a}$ (from 25.9 to $29.4 \%$ ) and a decrease in the prevalence of genotype $1 \mathrm{~b}$ (from $44 \%$ to $39,5 \%$ ) has been observed over time. Patients with genotype 4 were, mostly, men and with HIV-HCV coinfection.

Conclusions: The prevalence of genotype 4 in our geographical environment was significantly different than the national average which leads to the conclusion that the spread of this genotype was much slower than suggested.

Key words: Hepatitis C. Genotypes. Spreading.

Correspondencia:

María del Carmen Ramos-Sánchez.

Hospital Universitario Río Hortega.

Calle Dulzaina, 2

47012 Valladolid

Tfno: 983-420400 ext. Laboratorio

Correo electrónico: montealeku@gmail.com 


\section{INTRODUCCIÓN}

De acuerdo con datos de la Organización Mundial de la Salud el virus de la hepatitis $\mathrm{C}$ (VHC) es actualmente la causa más importante de enfermedad hepática crónica y muerte relacionada con el hígado y afecta a unos 175 millones de personas. La prevalencía varía mucho por países y regiones y oscila entre el $0,02 \%$ de Reino Unido o Finlandia al 18\% de Egipto ${ }^{1}$.

En las diferentes áreas geográficas se han obtenido secuencias que agrupan al virus en genotipos en función de su homología ( 1 a 6) y subtipos por orden de descubrimiento (a, b y c).

Según Echevarria et al, 2006², parece que dos epidemias separadas de VHC tuvieron lugar en España durante los últimos 30 años. La primera involucró la dispersión de los genotipos 1a y 3 , la segunda fue más reciente e involucró la diseminación del genotipo 4. Datos posteriores de estos y otros autores han sugerido que la prevalencia de las cepas VHC genotipo 4 se están extendiendo en la población española.

Adicionalmente al interés de tipo epidemiológico, la determinación del genotipo tiene importancia clínica al orientar sobre la respuesta potencial a la terapia y la duración de la misma: (i) los genotipos 1 y 4 responden menos a dicho tratamiento que los 2, 3, 5 y 6 y (ii) mientras la duración de la terapia estándar basada en el interferón para los genotipos 1 y 4 es de 48 semanas, para los genotipos 2 y 3 es solo de 24 semanas.

El objetivo del trabajo fue explorar la tendencia de dispersión de genotipos VHC a través del seguimiento de su prevalencia en la zona de influencia de nuestro hospital durante los periodos 1999-2002, 20032006 y $2007-2010$.

\section{MATERIAL Y MÉTODO}

El material primario para la realización del estudio fueron 1.074 muestras séricas extraídas en nuestro laboratorio a sujetos diagnosticados de infección por VHC. El $90 \%$ de ellos eran pacientes procedentes de consultas de atención primaria derivados a las consultas externas del Hospital Universitario Río Hortega de Valladolid. El medio geográfico de procedencia de los pacientes fue el área de salud Valladolid Oeste (ASVO), constituida por una población mayoritariamente de carácter urbano (correspondiente a los populosos barrios vallisoletanos de Delicias, Huerta del Rey y Parquesol y a localidades como Tordesillas y Laguna de Duero) y minoritariamente rural (Mayorga, Medina de Rioseco, Villafrechós, Villalón de Campos y Mota del Marqués).

La extracción de ARN del VHC se llevó a cabo mediante el sistema COBAS AmpliPrep (Roche). La ). En pacientes coinfectados VHC-VIH la prueba de anticuerpos anti-VIH se realizó por una técnica EIA y en caso de positividad fue confirmada porWestern Blot o LIA. La transcripción inversa, amplificación e hibridación inversa se realizaron con reactivos Qiagen y Siemens.

Para la realización de pruebas estadísticas se utilizó el programa SPSS, versión 15. El análisis de la varianza se consiguió aplicando la prueba F de Fisher-Snedecor y comparando los valores obtenidos en el estudio con los valores correspondientes en dicha prueba a partir de un nivel de confianza fijado y con unos grados de libertad determinados en función de los factores de estudio.

\section{RESULTADOS}

Nuestros resultados aparecen reflejados en las diversas columnas de la tabla 1 . Los relativos al período enero 1999-diciembre 2002, recogidos en la primera columna, son considerados como referencia para las comparaciones sucesivas. 
Tabla 1

Seguimiento de la prevalencia de los genotipos del virus de la hepatitis $\mathbf{C}$ en el área de salud Valladolid oeste (ASVO) durante un periodo de 12 años y relación con resultados de una estadística a nivel estatal

\begin{tabular}{|c|c|c|c|c|c|}
\hline $\begin{array}{l}\text { Genotipo } \\
\text { VHC }\end{array}$ & $\begin{array}{c}\text { ASVO } \\
1999-2002\end{array}$ & $\begin{array}{c}\text { ASVO } \\
2003-2006\end{array}$ & $\begin{array}{c}\text { ASVO } \\
2007-2010\end{array}$ & $\begin{array}{c}\text { ASVO Global } \\
1999-2010\end{array}$ & $\begin{array}{c}\text { Estatal }^{2} \\
1996-2004\end{array}$ \\
\hline & casos $/ \%$ & casos $/ \%$ & casos $/ \%$ & casos $/ \%$ & $\%$ \\
\hline $\begin{array}{l}1 \\
\text { la }\end{array}$ & $92 / 25,9$ & $118 / 27,9$ & $87 / 29,4$ & $297 / 27,7$ & 24,1 \\
\hline $1 \mathrm{~b}$ & $156 / 44$ & $171 / 40,4$ & $117 / 39,5$ & $444 / 41,3$ & 41,3 \\
\hline $2 \mathrm{a} / 2 \mathrm{c}$ & $10 / 2,8$ & $15 / 3,5$ & $7 / 2,3$ & $32 / 2,9$ & 3,1 \\
\hline $3 a$ & $70 / 19,7$ & $82 / 19,4$ & $59 / 19,9$ & $211 / 19,6$ & 19,6 \\
\hline 4 & $7 / 2,0$ & $13 / 3,1$ & $5 / 1,7$ & $25 / 2,3$ & \\
\hline $4 a$ & $2 / 0,6$ & & & & \\
\hline $4 c / 4 d$ & $16 / 4,4$ & $23 / 5,5$ & $21 / 7,1$ & $63 / 5,9$ & 11,6 \\
\hline $4 \mathrm{f}$ & $1 / 0,3$ & & & & \\
\hline $5 \mathrm{a}$ & $1 / 0,3$ & $1 / 0,2$ & $-/-$ & $2 / 0,2$ & 0,3 \\
\hline Total & $55 / 100$ & $23 / 100$ & $296 / 100$ & $1074 / 100$ & 100 \\
\hline
\end{tabular}

Para el periodo enero 2003-diciembre 2006 la prevalencia de los genotipos VHC en nuestra área de salud es mostrada en la segunda columna de la tabla. Se observó un aumento de un $1,3 \%$ en los genotipos 4 , hasta el 8,6\%.

Los datos del último cuatrienio, 20072010 (tercera columna de la tabla) mostraron el mantenimiento de la tendencia al aumento de los genotipos 4 (que llegaron a representar el $8,8 \%$ de la distribución), y la de los tipos 1 y 1 a (desde $25,9 \%$ a $29,4 \%$ ) compensada por una ligera pero progresiva disminución de los porcentajes del genotipo $1 \mathrm{~b}$ (desde $44 \%$ a $39,5 \%)$.

Por otra parte, la distribución global de genotipos VHC (cuarta columna de la tabla), representativa en cuanto al número de casos y años incluidos, resultó muy similar a la de la última media estatal salvo por el mayor porcentaje de genotipos 1 y 1 a $(27,7 \%)$ y el menor porcentaje de genotipos $4(8,2 \%)$.

Como prueba de significación estadística para analizar los cambios entre las diversas columnas de la tabla se aplicó el análisis de la varianza (ANOVA) de una sola vía, realizado a través de la prueba $\mathrm{F}$ de Fisher-Snedecor. A un nivel de confianza de $0,05 \mathrm{~F}$ proporcionó valores superiores al valor crítico $(0,233)$ cuando fueron comparados el segundo y tercer cuatrienios entre sí $(0,97)$ y cada uno de ellos con el periodo global $(0,95$ y 0,982$)$, lo que indica que las distribuciones de genotipado fueron significativamente diferentes en esos periodos. No obstante, ni las distribuciones del cuatrienio inicial ni las correspondientes al periodo global resultaron significativamente diferentes cuando se enfrentaron a la distribución observada a nivel estatal (F0,05 $=1,08$ 1,14 frente a valores críticos de 4,28).

El genotipo mayoritario en nuestra población fue el $1 \mathrm{~b}$, con un porcentaje global del 41,3\% (39,5-44\%).

En relación a los pacientes con genotipo 4 la realización de un análisis particular en aspectos de distribución por sexo y coinfección VIH-VHC llevó a establecer que el $85 \%$ eran varones y el $15 \%$, mujeres y que la coinfección VIH-VHC alcanzó al 58\%. 


\section{DISCUSIÓN}

Nuestros datos para el primer periodo considerado, 1999-2002 ${ }^{3}$ fueron acordes con los referidos para 17 regiones españolas (enero 1996 - diciembre 2004): 1a: 24,1\%; lb: $41,3 \% ; 2: 3,1 \% ; 3: 19,6 \%$; $4: 11,6 \%$; 5 : $0,3 \%^{2}$, salvo en la significativa menor prevalencia de los genotipos tipo 4 (7,3\%). Sucesivamente, la prevalencia de este genotipo 4 en nuestra área evolucionó desde el porcentaje $7,3 \%$ hasta porcentajes del $8,6 \%$ en el periodo 2003-2006, para terminar alcanzando, en los últimos 4 años el 8,8\% y aproximarse, aún más, a la media estatal $(11,6 \%)$. Tal progresión evidenció la dispersión prevista para el genotipo 4 pero a una velocidad relativamente lenta: la prevalencia reflejada en el último porcentaje siguió siendo significativamente diferente de la media estatal.

En relación con las características de los pacientes con genotipo 4 parece importante resaltar que fueron, mayoritariamente, de sexo masculino y coinfectados VIH-VHC.

Concerniente a otros genotipos, los cambios producidos más significativos fueron: aumento de prevalencia para los genotipos 1 y 1a; una disminución para el 1b; y una escasa o nula presencia de casos para el genotipo 5 (esta última, en excelente coincidencia con un hallazgo de Gomila-Sard et al para sujetos coinfectados VIH-VHC de la provincia de Castellón ${ }^{4}$ ). Del genotipo 3a puede afirmarse que su prevalencia se mantuvo muy conservada con el tiempo y globalmente igual al de la distribución estatal.

Respecto a los pacientes con genotipo $1 \mathrm{~b}$, mayoritarios tanto en nuestra población como en las otras estadísticas disponibles, cabe destacar que, aunque su prevalencia comenzó siendo del 44\%, globalmente resultó ser la misma que la media estatal $(41,3 \%)$, y bastante alejada de la informada para el área geográfica de El Ferrol (56,2\%) 5. Consideramos que estas diferencias de porcentajes fueron determinadas, al menos en parte, por la proporción de pacientes con historia de transfusiones incluidos en los estudios pues, como es sabido, estos pacientes exhiben casi en su totalidad el fenotipo $1 \mathrm{~b}$ y mientras en la población de El Ferrol representaron el $14 \%$, en nuestra población no llegaron al 7\%. Precisamente, una limitación de nuestro estudio fue la indeterminación sobre el grado de representatividad del colectivo minoritario con historia de transfusiones respecto de la población global.

La conclusión del estudio fue que al ser la prevalencia del genotipo 4 en el área oeste de Valladolid significativamente diferente de la media estatal, la dispersión de este genotipo está siendo mucho más lenta de lo previsto y que se precisará de estudios posteriores para evidenciar su evolución.

\section{BIBLIOGRAFÍA}

1. WHO. Hepatitis C-global prevalence (update). Wkly Epidemiol Rec 1999; 74:425-7.

2. Echevarría JM, León P, Pozo F, Avellón A. Follow-up of the prevalence of hepatitis $C$ virus genotype in Spain during a nine year period (1996-2004). Enferm Infecc Microbiol Clin. 2006; 24:20-5.

3. Ramos-Sánchez MC, Torío-Cabezon R, MazónRamos MA, Martin-Gil FJ, Álamo M. Hepatitis C virus genotype 4 in a Northwest Spain district. J Clin Virol. 2005; 28:223-4

4. Gomila-Sard B, Amselem-Albuixech L, PardoSerrano FJ, Moreno-Muñoz R, Celades-Porcar E, Pontón Moreno JM. Estudio de la prevalencia de los genotipos del virus de la hepatitis $\mathrm{C}$ en la provincia de Castellón. Enferm Infecc Microbiol Clin. 2004; 22:130137 .

5. Touceda S, Pereira M, Agulla A. Prevalencia de genotipos del virus de la hepatitis $\mathrm{C}$ en el área de $\mathrm{El}$ Ferrol (La Coruña). Enferm Infecc Microbiol Clin. 2002;20(5):200-4 